\title{
Perspectives of Ramsey Schemes Based on High-Order Harmonics for High-Resolution XUV Spectroscopy ${ }^{1}$
}

\author{
A. Pirri ${ }^{a}$, E. Sali ${ }^{a}, b, *$, C. Corsi ${ }^{a}$, M. Bellinia ${ }^{a, c}$, S. Cavalieri ${ }^{a, b}$, and R. Eramo ${ }^{a, b, c}$ \\ ${ }^{a}$ European Laboratory for Non-Linear Spectroscopy (LENS), Università di Firenze, \\ Via N. Carrara 1, I-50019 Sesto Fiorentino, Firenze, Italy \\ ${ }^{b}$ Dipartimento di Fisica and CNISM, Università di Firenze, Via G. Sansone 1, I-50019 Sesto Fiorentino, Firenze, Italy \\ ${ }^{c}$ Istituto Nazionale di Ottica Applicata (CNR), Largo E. Fermi 6, I-50125 Firenze, Italy \\ *e-mail: sali@fi.infn.it \\ Received October 30, 2009; in final form, November 9, 2009; published online April 2, 2010
}

\begin{abstract}
We present an experimental and theoretical investigation of Ramsey-type interference fringes using high-order harmonic generation. The ninth harmonic $(\lambda \simeq 88 \mathrm{~nm})$ of a femtosecond Ti:sapphire laser was used to excite a pair of autoionizing states of krypton. The outcome of the ionization process, detected by an ion-mass spectrometer, shows the characteristic quantum interference pattern. The behavior of the fringe contrast was interpreted on the basis of a simple analytical model, which reproduced well the experimental results without any free parameter. Finally, we also present a proposal for a similar experiment to be performed on high-lying bound state of atomic argon, with the aim of achieving even higher resolving power.
\end{abstract}

DOI: $10.1134 / \mathrm{S} 1054660 \mathrm{X} 10090215$

\section{INTRODUCTION}

Consistent scientific efforts have been devoted in recent years towards achieving high spectroscopic resolution in the vacuum ultraviolet (VUV) and extreme ultraviolet (XUV) regions, where important investigations involving several atomic and molecular species can be performed [1-5]. A broad and simple tunability in all this spectral range and extending into the soft-X rays is only achieved by synchrotron facilities, which, when used in combination with the best monochromators available, can provide radiation with a bandwidth of the order of $0.5-1.0 \mathrm{~cm}^{-1}$ [6]. Apart from their limitations in spectral resolution, synchrotron sources suffer from not being user-friendly: indeed, table-top-size sources would be much more desirable for a more widespread use. On the other hand, laserbased narrowband $\mathrm{cw}$ sources hardly exist at such short wavelengths [7].

However, the situation has been rapidly evolving in recent years and several experiments have shown that it is possible to obtain a high spectral resolution at short wavelengths. This was made possible by the development of mode-locked lasers of femtosecond duration which, when interacting in a non-resonant way with noble gas atoms, can extend the generated spectrum much further into the XUV and soft X-ray regions through the process of high-order harmonic generation (HOHG). In fact, this technique would be inherently affected by a broad bandwidth of the generating femtosecond pulse. However, recent studies have shown that high-resolution can be obtained with systems based on HOHG. The general idea is similar to

\footnotetext{
1 The article is published in the original.
}

what was first introduced by Ramsey in 1950 [8] to face the transit-time-limited interaction between the molecules in a jet and a microwave field. In that case a greater effective interaction time was obtained by using two widely-spaced interaction zones. In an analogous way one may think to extend the interrogation time characteristic of an ultra-short harmonic field by using pairs (or sequences) of collinear, phase-coherent, and time-delayed pulses [9-12]. In a very simple representation of this idea, a first short pulse may be thought of as inducing a coherence in a two-level system, creating a dynamical polarization of the medium. The induced polarization then oscillates at the transition frequency with an amplitude that decays on the timescale of its characteristic dephasing time. Depending on the phase delay of the second pulse, this may either further excite the system or bring it back to the ground state. As a result, any excitation-related observable exhibits interference fringes when the delay between the two pulses is varied. Important spectroscopic information such as the absolute frequency of the transition, the dephasing time, or the energy separation of nearby states can be extracted with this method.

In order to extend this technique to high-order harmonics in the XUV it is necessary to transfer the step of pulse splitting and delaying from the XUV pulse to the harmonic-generating beam, and relying on the phase coherence of the generation process [13-16]. The first experimental demonstration of Ramsey-type spectroscopy with high-order harmonics was presented by our group a few years ago [4]. In that experiment a couple of autoionizing states of krypton were excited by a pair of pulses at $\lambda \simeq 88 \mathrm{~nm}$, the ninth har- 


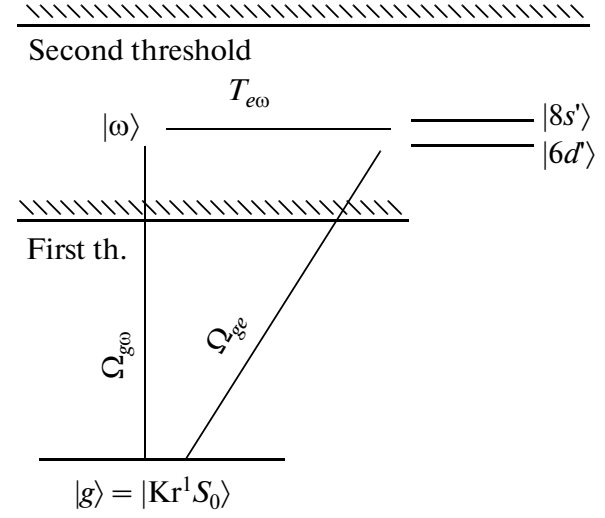

Fig. 1 Scheme of the interaction, showing the involved autoionizing levels of the krypton atom. $\Omega_{g e}$ is the Rabi frequency for the coupling between the ground state $|g\rangle$ and each autoionizing state $|e\rangle$; as explained in the text, we are working in a perturbative regime and a simple two-state dynamics involving ground state and a single autoionizing state has to be taken into account. $\Omega_{g \omega}$ is the analogue for the continuum states $|\omega\rangle$. The coupling between the autoionizing state and the continuum, due to an intraatomic interaction, has been denoted by $T_{e \omega}$.

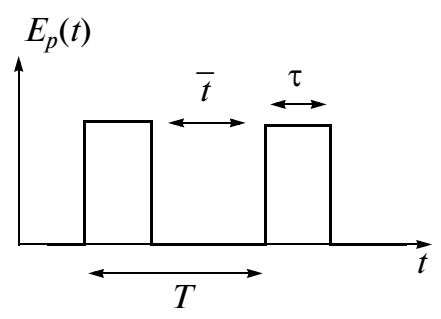

Fig. 2. Temporal scheme of the pulse pair as treated in the theory, i.e., flat top, duration $\tau$, delay $T$, separation $\bar{t}=$ $T-\tau$.

monic of a Ti:sapphire laser. The observation of quantum interferences in the ionization signal allowed us to extract useful spectroscopic information.

In this paper we present an extension of our previous experimental study of Ramsey-type spectroscopy [4] using the same combination of laser frequency and atomic levels. Compared to our previous work, in this study several changes were introduced in order to make the experimental results and their comparison with theory more robust. Besides a general improvement of the experimental setup, which allowed us to perform the experiments exploiting the full repetition rate of our laser, the most important change is the choice of collecting ions instead of electrons, which allows a comparison between theory and experiment free of unknown parameters. Also, as far as the theoretical model, an analytical solution was found that is able to reproduce satisfactorily the experimental data with no unknown parameters. Finally, we also present a proposal for a similar experiment to be performed on high-lying bound state of atomic argon, with the aim of achieving even higher resolving power.

The paper is organized as follows. In Section 2 we present the theory at the basis of the experiments and the calculations, namely the two-state theory with some analytical results and the calculation performed in the actual case for the comparison with the experimental data. In Section 3 we describe the experimental setup, with particular emphasis on the main novelties introduced in this work with respect to our previous one. In Section 4 we present the experimental results and the comparison with theoretical calculations. In Section 5 we present our proposal for the experiment on bound states. Finally, in Section 6 a brief summary is presented.

\section{THEORETICAL MODEL}

In this section we present the theoretical model at the basis of our work [17]. Figure 1 shows a scheme of the levels and laser fields involved. We work in a perturbative regime and a simple two-state dynamics involving ground state and a single autoionizing state is taken into account. Our aim is to obtain an analytical model for the dependence of the fringe contrast upon the pulse delay. Figure 2 shows the temporal scheme of the pulse pair as treated in the theory. In a two-state approach, the atomic system ground state $|g\rangle$ is coupled to the autoionizing state $|e\rangle$ by means of a field at frequency $\omega_{p}$ (see Fig. 1). We start from the atomic state vector expansion

$$
|\Psi\rangle=c_{g}(t)|g\rangle+c_{e}(t)|e\rangle+\int d \omega c_{\omega}|\omega\rangle
$$

in terms of the bound and continuum states. We work in the interaction picture, and use the rotating wave approximation and the usual procedures of Markov elimination for the continuum amplitude to get

$$
\begin{aligned}
& \left(\frac{d}{d t}+\frac{\Gamma_{g}}{2}\right) c_{g}=\frac{i \gamma}{2}(-q+i) e^{i \Delta t} c_{e}, \\
& \left(\frac{d}{d t}+\frac{\Gamma_{e}}{2}\right) c_{e}=\frac{i \gamma}{2}(-q+i) e^{-i \Delta t} c_{g} .
\end{aligned}
$$

In these equations, the notations

$$
\begin{gathered}
\Gamma_{g}=\left(\pi \frac{\Omega_{g \omega} \Omega_{\omega g}}{2}\right)_{\omega=\omega_{0}} \Gamma_{e}=2 \pi\left[T_{e \omega} T_{\omega e}\right]_{\omega=\omega_{0}}, \\
\gamma=\pi\left(\Omega_{g \omega} T_{\omega e}\right)_{\omega=\omega_{0}}=\sqrt{\Gamma_{g} \Gamma_{e}}, \\
q=\frac{f d \omega \frac{\Omega_{g \omega} T_{\omega e}}{\omega-\omega_{0}}+\Omega_{g e}}{\pi\left(\Omega_{g \omega} T_{\omega e}\right)_{\omega=\omega_{0}}}, \quad \Delta=\omega_{g}+\omega_{p}-\omega_{e}
\end{gathered}
$$

have been used for the decay constants $\Gamma_{g}$ and $\Gamma_{e}$, the coupling constant $\gamma$, the Fano parameter $q$, and the detuning $\Delta$. In these definitions $\omega_{0}=\omega_{g}+\omega_{p} \approx \omega_{e}$ is 
the frequency in the continuum where the laser is tuned, $\Omega_{\omega k}=E_{p}\langle\omega|\mathbf{d} \cdot \hat{\mathbf{e}}| k\rangle / \hbar(k=g, e)$ are the Rabi frequencies,

$$
\mathbf{E}=E_{p} \hat{\mathbf{e}} \cos \omega_{p} t
$$

is the (linearly polarized) probe field and $T_{\omega k}$ are the matrix elements of the autoionizing interaction. In (2), (3), the dynamic and static Stark shifts $\delta \omega_{g}$, and $\delta \omega_{e}$ are thought as included in the detuning.

An explicit analytical result for the ionization probability $I$ can be obtained assuming two identical pulses of duration $\tau$ and delay $T$, with $T \geq \tau$ and a resonant interaction, i.e., $\Delta \tau \approx 0$. Following [3] one can obtain $c_{g}^{(k)}$ and $c_{e}^{(k)}$, i.e., the parts of the amplitudes which are of the order of the Rabi frequency $\left(\Omega_{g \omega}\right)^{k}$. In this way, the ionization probability, $I=1-\left|c_{g}(T+\tau)\right|^{2}$ is given by

$$
I=2 I_{1}+\left[q^{2}+1\right] I_{f} e^{-\Gamma_{e}(T-\tau) / 2} \cos \left(v+\omega_{e g} T\right),
$$

where

$$
\tan v=\frac{2 q}{q^{2}-1} .
$$

In the limit $T \longrightarrow \infty$, we recognize from Eq. (4) the ionization probability of the single pulse multiplied by 2. If we also assume flat-top pulses (as in Fig. 2) the expressions of $I_{f}$ and $I_{1}$ can be written as:

$$
\begin{gathered}
I_{f}=\frac{2 \Gamma_{g}}{\Gamma_{e}}\left(1-e^{-\Gamma_{e} \tau / 2}\right)^{2}, \\
I_{1}=\Gamma_{g} \tau+\left(q^{2}-1\right) \Gamma_{g}\left[\tau+\frac{2}{\Gamma_{e}}\left(e^{-\Gamma_{e} \tau / 2}-1\right)\right] .
\end{gathered}
$$

We can now define the following two quantities, i.e. the maxima and minima of the ionization yield $I$ as a function of the separation $\bar{t}=T-\tau$ between the two pulses:

$$
I_{ \pm}=2 I_{1} \pm\left(q^{2}+1\right) I_{f} e^{-\Gamma_{e} \bar{t} / 2},
$$

and from these we can write the fringe contrast as

$$
\rho=\frac{I_{+}-I_{-}}{I_{+}+I_{-}}=\frac{\left(q^{2}+1\right)\left(1-e^{-\Gamma_{e} \tau / 2}\right)^{2} e^{-\Gamma_{e} \bar{t} / 2}}{\Gamma_{e} \tau+\left(q^{2}-1\right) \Gamma_{e}\left[\tau+\frac{2}{\Gamma_{e}}\left(e^{-\Gamma_{e} \tau / 2}-1\right)\right](9)} .
$$

Expression (9) was obtained for one autoionizing state only and assuming resonant excitation (detuning $\Delta=$ 0 ). In order to have a theoretical quantity that can provide a meaningful comparison with our experimental data, it is necessary for our model to include a detuning $\Delta \neq 0$ and the presence of a second autoionizing state. The introduction of the detuning leads to a slightly more complicated expression for the ionization yield, whose complete form can be found in [17].
As for the presence of the second state, so long as we work in a perturbative regime, no coupling exists between the two autoionizing states, which thus evolve independently following a two-state dynamic. In our experiment, as schematically shown in Fig. 1, the two excited autoionizing states of krypton that we are interested in are the $4 p^{5}\left({ }^{2} P_{1 / 2}\right) 6 d^{\prime}$ and $4 p^{5}\left({ }^{2} P_{1 / 2}\right) 8 s^{\prime}$. They have an energy separation of about $29 \mathrm{meV}$, well below the $0.21 \mathrm{eV}$ of the single-pulse spectral width. Both of them are thus simultaneously excited by the ninth-harmonic of our laser at $88.2 \mathrm{~nm}$. The theoretical ionization probability including both states is then simply obtained by summing up the independent contributions of two single states with proper choice of the values of energy and detuning. As a consequence of the difference in energy between the two states, a beating should be visible in a plot of the fringe contrast of the ionization signal as a function of the delay between the pulses. In the present situation, where the lifetimes of the two states are of different order of magnitude ( 461 fs for the $8 s^{\prime}$ and 26 fs for the $6 d^{\prime}$ ) the "delay window" where the beating is observable is determined by the equality between the ion modulations of the two states. The period of the beating is determined by the difference in the energy of the two states. Indeed, a beating is clearly visible in the curves of Fig. 6 .

To summarize, in our analytical model we assume the harmonic field to be made of a pair of flat-top pulses, with a FWHM duration of 17 fs derived from the measured $1.4 \mathrm{~nm}$ linewidth of the single-pulse harmonic spectrum. The parameters that we introduce in the model, i.e., the state energy, energy widths, ionization constants and the Fano parameters, are those reported in the synchrotron measurement of [18]. In our experiment we chose to measure the ion signal instead of electrons, which implied that no parameter remains unknown. This allows a more strict comparison with the experimental results, as will be extensively discussed in the next sections.

\section{THE EXPERIMENTAL SETUP}

Figure 3 shows schematically the experimental apparatus, which is composed basically of four units (plus the laser system): the Michelson interferometer, the harmonic generation chamber, the XUV monochromator and the interaction chamber. Each of these stages presents some improvements or changes with respect to the previous work. The light source is an amplified Ti:sapphire laser delivering pulses with up to $\sim 1 \mathrm{~mJ}$ energy per pulse, $\sim 30$ fs time duration at a repetition rate of $1 \mathrm{kHz}$, with central wavelength set at $793.7 \mathrm{~nm}$. The laser pulse passes through a Michelson interferometer, which divides the fundamental pulse into two replicas by means of a 50/50 beam splitter. The relative delay between the two pulse replicas can be varied by translating the folding mirror in one arm of the interferometer. Long translations are obtained using a computer-controlled stepping motor, while 


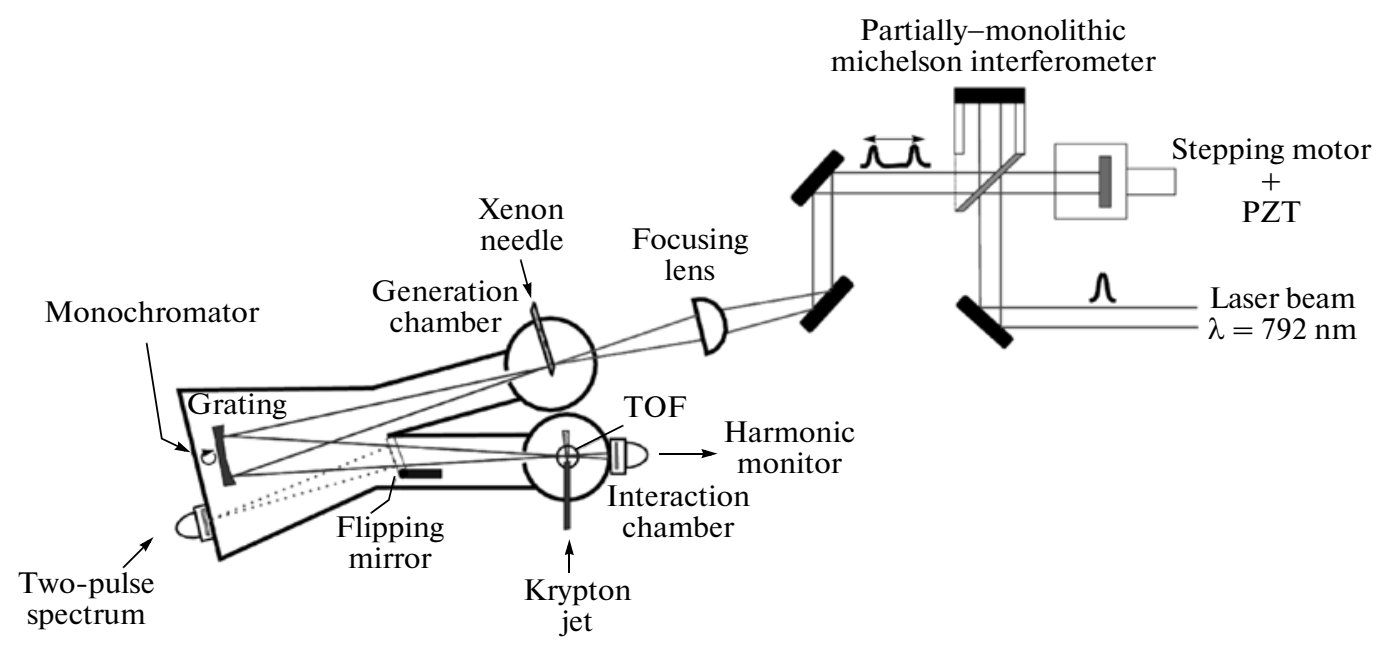

Fig. 3. Experimental set-up. The ninth harmonic, generated by focusing the phase-locked laser pulse pair produced by the Michelson interferometer in the xenon flow and selected by the XUV monochromator, is focused in the krypton jet in the interaction chamber. The ions ejected in the one-photon ionization process are energy-analyzed by a TOF spectrometer. A flipping mirror can redirect the harmonic field to the exit slit of the monochromator, where a spectrum of the two-pulse field can be measured and used for the fine alignment of the Michelson interferometer. A phosphor/phototube pair, at the exit of the interaction chamber, monitors the transmitted harmonic field intensity.

shorter scans are obtained by applying a triangularwave voltage to a piezoelectric crystal that holds the mirror. Great attention was devoted to reducing as much as possible any possible source of mechanical instabilities in order to increase the accuracy of the measurement. A partially-monolithic quartz interferometer was chosen and the whole device was covered by a Plexiglas box and isolated from the optical table by means of anti-vibration plastic sheets. Mechanical and thermal drifts around $10-20 \mathrm{~nm} / \mathrm{min}$ were experimentally estimated.

The laser beam is focused in the first vacuum chamber by a $25-\mathrm{cm}$ focal-length plane-convex lens.

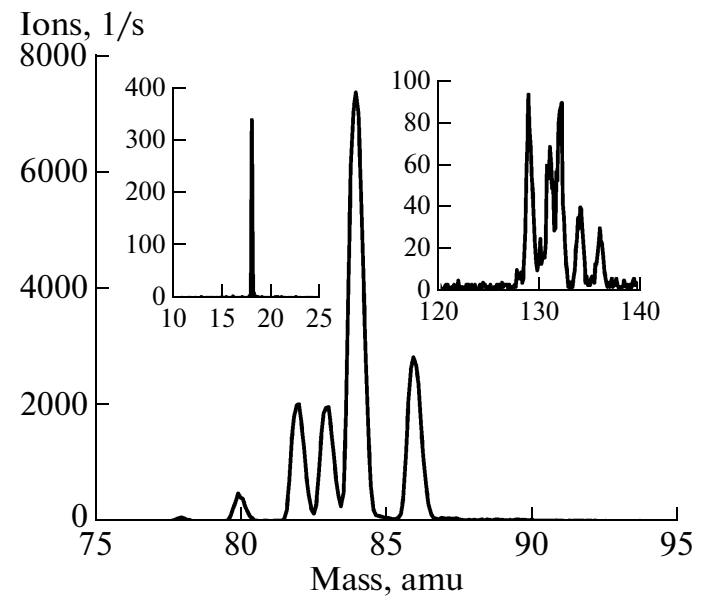

Fig. 4. Typical ion mass spectrum. Besides the krypton ions, the spectrum shows the presence of water (top-left inset) and xenon (top-right inset) coming from the harmonic generation chamber
An intensity of about $5 \times 10^{14} \mathrm{~W} / \mathrm{cm}^{2}$ is reached in the focal spot, where a steel capillary containing xenon is placed. The laser itself drills two holes in the capillary (entrance and exit) with a diameter of approximately $50 \mu \mathrm{m}$ each. The gas flows continuously through these holes, allowing for a temporally constant xenon pressure profile in the spot where harmonics are generated. The generated harmonic radiation enters the XUV monochromator, where it is spectrally separated and re-focused by a $\mathrm{Pt}-\mathrm{Ir}$ normal-incidence spherical grating (600 lines $/ \mathrm{mm}$ ), which selects for our purpose the 9th harmonic, corresponding to a wavelength of $88.2 \mathrm{~nm}$. The refocused harmonic then interacts with

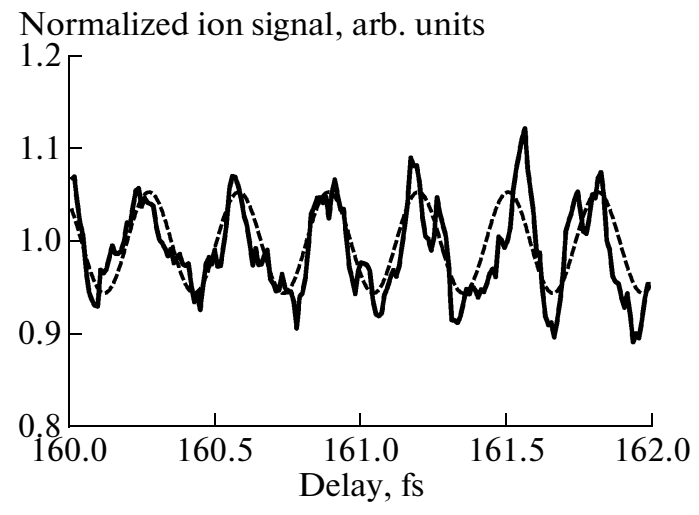

Fig. 5. Detail of the fringe pattern in the normalized ion signal (continuous curve) around a delay $\Delta t=160$ fs. A sinusoidal fit (dashed curve) is superimposed. The fringe spacing corresponds to the 0.29 fs-period of the atomic transition. Experimental points result from summing over 1200 laser pulses and smoothing on a fraction $(\approx 20 \%)$ of the fringe period. 
the krypton atoms inside an ion-mass time-of-flight (TOF) spectrometer, which replaces the exit slit of the monochromator (more details about the interaction region are provided towards the end of this section).

The TOF spectrometer is equipped with three molybdenum disks where DC extraction fields up to $250 \mathrm{~V} / \mathrm{cm}$ are applied for efficient charge collection. The interaction region is placed between the first two plates, where a continuous flow of krypton atoms is injected by means of a needle, perpendicularly to the TOF axis and to the harmonic field propagation. The charges leave the interaction volume through a 2-mm diameter aperture on the second and third molybdenum plates, and enter a field-free zone. The flight tube is a $35-\mathrm{cm}$ long, gold-plated, copper cylinder with a diameter of $5 \mathrm{~cm}$. In order to prevent external magnetic field effects, a coaxial $\mu$-metal cylinder encloses both the flight tube and the interaction region. A microchannel plate assembly placed at the end of the field-free zone detects the ions. The time-resolved signal is amplified and shaped by a fast constant fraction discriminator and sent to a fast multiscaler PC board with $\sim 0.5 \mathrm{~ns}$ time resolution. Finally it is stored in a computer and analyzed.

A very critical element for the experiment is the collinear alignment of the two harmonic beams. For this aim, a first check is done on the alignment of the two infrared beams coming out of the interferometer, by visually inspecting (using an infrared-sensitive card) the optical interference fringes that they produce. Fine alignment is performed by means of three piezoelectric crystals acting on the kinematic mounts of the folding mirror in one arm of the interferometer. During the measurements a similar check is kept 'online' using a $\mathrm{He}-\mathrm{Ne}$ laser beam that enters the interferometer collinearly with the infrared laser and checking the fringes on the $\mathrm{He}-\mathrm{Ne}$ profile. This check allows us to control the alignment of the two infrared pulses by monitoring pulse front displacements of the order of hundreds of nm over the beam diameter. Once the two infrared beams are aligned to the best of our capabilities, a second, more stringent alignment check is done directly on the harmonic beams. In fact, after being separated by the grating, the harmonics can be either directed into the interaction chamber, or deviated by a flipping mirror towards an exit slit at the back of the monochromator (see Fig. 3), where they can be detected by means of a phosphor screen and a photomultiplier tube. By measuring the two-pulse spectrum and by maximizing the fringe contrast it is possible to optimize the horizontal alignment of the two harmonic beams on the scale of a fraction of the harmonic wavelength as shown in [16].

Compared to our previous study [4] in this work we decided to detect ions instead of electrons as a product of the ionization. At that time, the main reason for that choice was essentially that in principle the electron spectra could provide a more stringent monitor over

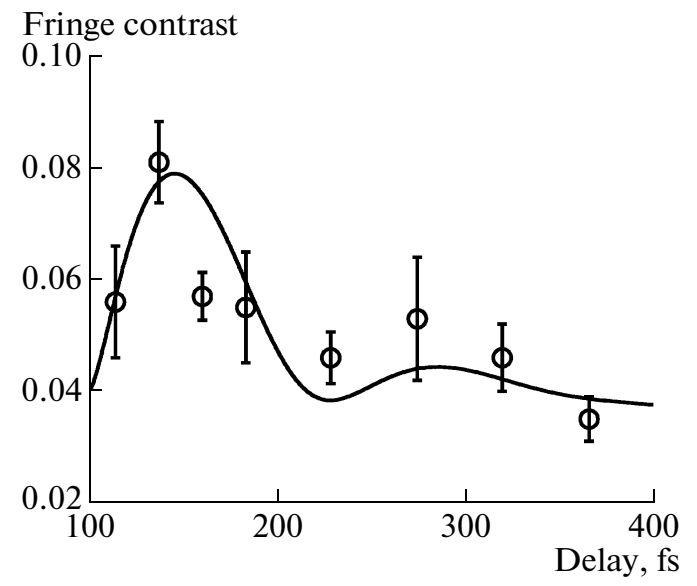

Fig. 6. Fringe contrast vs. pulse delay. The solid curve superimposed to the data points is a theoretical curve, as explained in the text, where atomic parameters are obtained by the synchrotron radiation measurements of [18]. No free parameter has been used in the theoretical curve.

the resonance condition. As a matter of fact, in the end this request did not turn out to be essential in the analysis of the results. On the other hand, the choice of detecting electrons implied that a parameter to be considered was the branching ratio between the differential ionization cross-sections of the $8 s^{\prime}$ and $6 d^{\prime}$ states along the direction of detection. This parameter is not known from the literature, therefore, in the fit of experimental data in [4], it was left as a free parameter. On the contrary, this parameter does not exist if one detects ions instead of electrons, since the higher mass of ions implies that effectively they can be considered as motionless immediately after ionization. For this reason, the comparison of experimental and theoretical results that is presented in this study represents a much more stringent test, since no unknown parameter is left free.

In Fig. 4 a typical ion spectrum is presented. It can be seen that the different isotopes are clearly resolved in the spectrum, implying a mass resolution better than $1 \mathrm{amu}$. This is due to the fact that, choosing the secondary focus of the spectrometer, the dimension of the beam along the TOF axis is so small $(30 \mu \mathrm{m})$ that we keep the advantage of a 2D collecting geometry. The choice of the primary focus would have not allowed this level of mass resolution, as the dimension of the beam along the time of flight spectrometer axis would have been too large. As a drawback, the $3 \mathrm{~mm}$ size in the horizontal direction implies a slightly reduced collection efficiency, since particle collection of our TOF is only efficient over an area of diameter of about $1 \mathrm{~mm}$. On the other hand, this reduction of collection volume results in an advantage as far as the ease of wavefront superposition is concerned. 


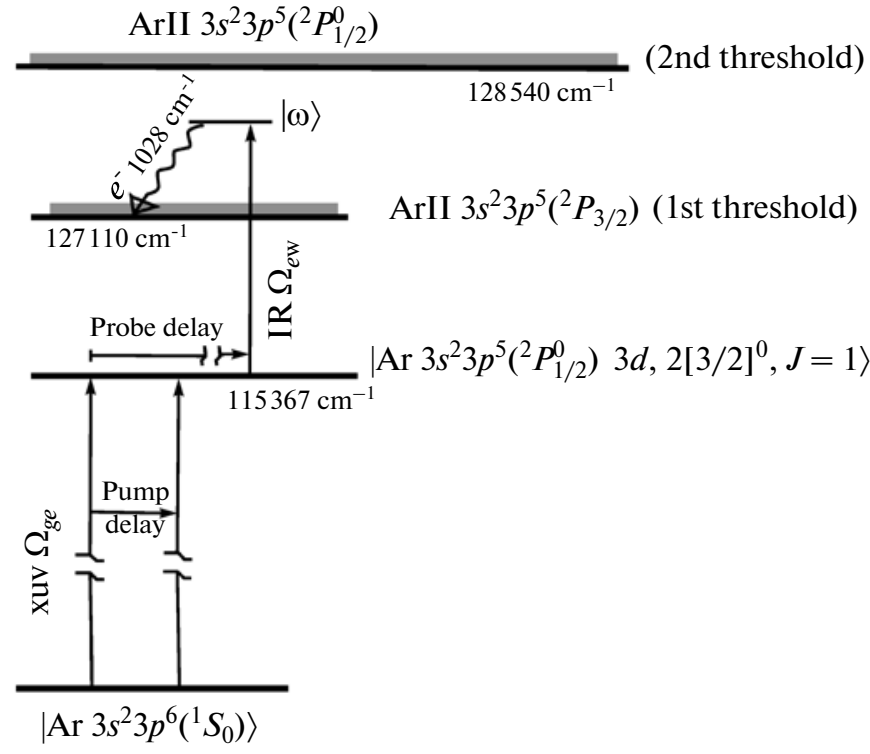

Fig. 7. Scheme of the interaction, showing the involved high-lying bound states of argon. $\Omega_{g e}$ are the probing harmonic fields, while $\Omega_{e \omega}$ is the portion of the infrared field that provides the ionization.

\section{RESULTS}

According to the theory (see Section 2) the quantum evolution of the system reveals itself as a fringe pattern in the ion yield when the delay between the two harmonic pulses is varied. Figure 5 shows a typical fringe pattern measured around a delay $\Delta t \sim 160 \mathrm{fs}$. During each measurement, all interesting signals are simultaneously acquired, i.e., the ions signal, the laser signals and the transmitted harmonic intensity as a reference monitor. Each point of the continuous curve corresponds to the integrated signal of all ion peaks in the krypton spectrum, divided by the reference monitor. In the same figure a sinusoidal fit is superimposed to the experimental data, giving a measure of the fringe visibility. We notice the characteristic Ramsey modulation, with a period given by $2 \pi / \omega_{e} \approx T_{f} / 9 \simeq 0.29 \mathrm{fs}, T_{f}$ being the period of the fundamental laser beam.

The fringe contrast (open circles), versus the delay between the two XUV-pulses is shown in Fig.6. The solid curve is the theoretical calculation, obtained assuming flat-top pulses (see section II). Precisely, the curve is obtained by summing up two contributions to take into account both states $8 s^{\prime}$ and $6 d^{\prime}$ of krypton. The agreement between the theoretical curve and the experimental data is quite good. The beating due to the energy difference between the two states, which is expected from the theory (continuous curve), can also be seen from the experimental results. As explained in Section 2 , the beating is observable only in a delay window where the ion modulations of the two states are comparable. This corresponds to a delay interval between 100 and 400 fs, i.e., intermediate between the

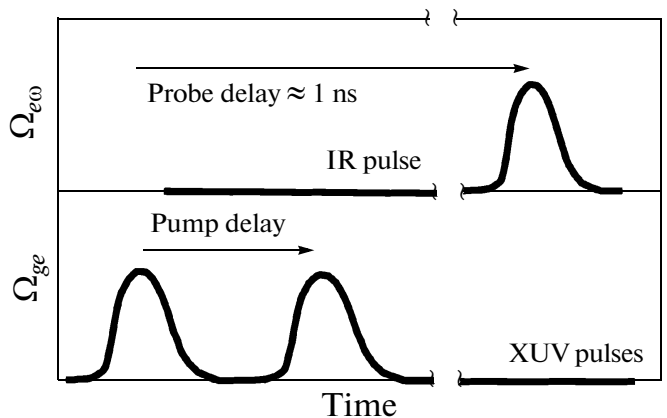

Fig. 8. Temporal scheme of the laser pulses as required in the bound-states argon experiment. $\Omega_{g e}$ : probing harmonic pulses. $\Omega_{e \omega}$ : infrared pulse that provides the ionization.

lifetimes of the two states. As already mentioned earlier in the text, the theoretical curve is obtained without any free parameter (see Section 2). In this respect, as far as the robustness of the comparison there is considerable difference between what is shown here in Fig. 6 and the similar figure in [4]; in that case one unknown parameter (the branching ratio between the differential ionization cross-sections of the $8 s^{\prime}$ and $6 d^{\prime}$ states along the direction of detection) was left as a fit parameter, whereas here all parameters are known from the literature [18].

\section{BOUND STATES}

In this last section we propose an evolution of the experiment reported above where the object of the study will be high-lying bound atomic states instead of autoionizing states lying in the continuum. With bound states level lifetimes are much longer (and the corresponding transition linewidths much narrower, although limited by Doppler broadening) and thus allow us to put to a stringent test the spectral resolution limits of the XUV Ramsey technique. A similar experiment, with a different way to produce the controlled delay between the pump pulses, has been recently used for the spectroscopy of a bound state at $125 \mathrm{~nm}$ in xenon and for a two-photon transition at $212 \mathrm{~nm}$ in krypton $[1,2]$. We propose the investigation of a single-photon XUV transition at $87 \mathrm{~nm}$ in atomic argon. The upper $3 s^{2} 3 p^{5}\left({ }^{2} P_{1 / 2}\right) 3 d$ level lies close to the first ionization threshold of the atom and excited electrons can be promoted to the continuum by a further absorption of a single IR photon at the fundamental wavelength. The relevant scheme of levels and laser fields is shown in Fig. 7, while Fig. 8 represents the temporal distances between the involved laser pulses. The necessity to operate in a XUV-pump/IR-probe setup will require a slight modification to the experimental setup shown in Fig. 3, since a part of the fundamental IR light beam is extracted (using a beamsplitter) and used to photoionize the excited argon 
atoms. Although some technical difficulties will have to be overcome, it is reasonable to assume that the proposed setup can be scaled up to delays in the range of tens of nanoseconds (corresponding to arm displacements in the meter range) to achieve a resolving power of $10^{8}$ in the XUV region.

\section{CONCLUSIONS}

Our experiment showed how the Ramsey-type technique, in conjunction with high-order harmonic generation, can be used as an efficient tool for highresolution spectroscopic investigations in the XUV. The results of the experiment, performed on krypton atoms in resonance condition with a couple of autoionizing states, show a clear quantum interference in the ions signal and a beat between the doublet ion components. These quantum fringes are detected as a function of the delay between the two harmonic pulses. Compared to a previous study that we performed on the same subject, we chose a configuration of ion-detection instead of electron-detection. This choice allows us to theoretically model the process without any unknown parameter left free, and the resulting model reproduces well the experimental data. We found that the main experimental limitation for a user-friendly spectroscopic application of this technique lies in the stability of the Michelson interferometer and in the wave front superposition of the two harmonic fields. We suggest that this last aspect can be overcome by detecting the signal from a smaller part of the volume of interaction between harmonic radiation and atomic samples. Finally, we proposed an evolution of the reported experiment where the object of the study will be high-lying bound atomic states instead of autoionizing states lying in the continuum, with the aim to achieve a resolving power of $10^{8}$ in the XUV region.

\section{ACKNOWLEDGMENTS}

This research has been performed at LENS. We acknowledge Riccardo Ballerini and Ahmed Hajeb for the accurate mechanical realizations, and Mauro Giuntini, Marco De Pas, and Alessio Montori for providing their assistance in the set-up of the electronics. This work was partially supported by Ente Cassa di
Risparmio di Firenze and by the EC's FP7 under grant agreement no. 228334 (JRA1: ALADIN).

\section{REFERENCES}

1. S. Witte, R. Th. Zinkstok, W. Ubachs, W. Hogervorst, and K. S. D. Eikema, Science 307, 400 (2005).

2. R. Th. Zinkstok, S. Witte, W. Ubachs, W. Hogervorst, and K. S. E. Eikema, Phys. Rev. A 73, 061801(R) (2006).

3. S. Cavalieri and R. Eramo, Phys. Rev. A 58, R4263 (1998).

4. S. Cavalieri, R. Eramo, M. Materazzi, Ch. Corsi, and M. Bellini, Phys. Rev. Lett. 89, 133002 (2002).

5. M. Kovacev, S. V. Fomichev, E. Priori, Y. Mairesse, H. Merdji, P. Monchicourt, P. Breger, J. Norin, A. Persson, A. L'Huillier, C.-G. Wahlstrom, B. Carre, and P. Saliéres, Phys. Rev. Lett. 95, 223903 (2005).

6. P. A. Heinmann, M. Koike, C. W. Hsu, D. Blank, X. M. Yang, A. G. Suits, and Y. T. Lee, Rev. Sci. Instrum. 68, 1945 (1997).

7. K. S. E. Eikema, J. Walz, and T. W. Hansch, Phys. Rev. Lett. 86, 5679 (2001).

8. N. F. Ramsey, Phys. Rev. 78, 695 (1950).

9. M. M. Salour, Rev. Mod. Phys. 50, 667 (1978).

10. J. T. Fourkas, W. L. Wilson, G. Wackerle, A. D. Frost, and M. D. Fayer, J. Opt. Soc. Am. 6, 1905 (1989).

11. M. Bellini, A. Bartoli, and T. W. Hansch, Opt. Lett. 22, 540 (1997).

12. R. van Leeuwen, M. L. Bajema, and R. R. Jones, Phys. Rev. Lett. 82, 2852 (1999).

13. R. Zerne, C. Altucci, M. Bellini, M. B. Gaarde, T. W. Hänsch, A. L'Huillier, C. Lyngå, and C. G. Wahlström, Phys. Rev. Lett. 79, 1006 (1997).

14. M. Bellini, C. Lyngå, A. Tozzi, M. B. Gaarde, T. W. Hänsch, A. L'Huillier, and C. G. Wahlström, Phys. Rev. Lett. 81, 297 (1998).

15. P. Salières, L. Le Deroff, T. Auguste, P. Monot, P. d'Oliveira, D. Campo, J. F. Hergott, H. Merdji, and B. Carre, Phys. Rev. Lett. 83, 5483 (1999).

16. M. Bellini, S. Cavalieri, C. Corsi, and M. Materazzi, Opt. Lett. 26, 1010 (2001).

17. A. Pirri, E. Sali, C. Corsi, M. Bellini, S. Cavalieri, and R. Eramo, Phys. Rev. A 78, 043410 (2008).

18. J. Z. Wu, S. B. Whitfield, C. D. Caldwell, M. O. Krause, P. van der Meulen, and A. Fahlman, Phys. Rev. A 42, 1350 (1990). 